\title{
Coriolis Interactions in Benzene-Water and Related Molecular Complexes
}

\author{
Prasenjit Halder ${ }^{\mathrm{a}}$, Mangala Sunder Krishnan ${ }^{\mathrm{a}, *}$, E. Arunan ${ }^{\mathrm{b}, *}$ \\ ${ }^{a}$ Department of Chemistry, Indian Institute of Technology Madras, Chennai 600 036, India \\ ${ }^{b}$ Department of Inorganic and Physical Chemistry, Indian Institute of Science, Bangalore \\ 560 012, India
}

Supplementary Material :

Table S1 : Vibrational frequencies from ab initio calculations for benzenewater and rare gas-benzene-water complexes at MP2/6-311++g(d,p) level, including BSSE CP (basis-set superposition error counterpoise) corrections

Table S2 : Ab initio coordinates of the equilibrium structure of $\mathrm{C}_{6} \mathrm{H}_{6}-\mathrm{H}_{2} \mathrm{O}$ complex at MP2 $/ 6-311++\mathrm{g}(\mathrm{d}, \mathrm{p})$ level, including BSSE CP corrections

Table S3 : Ab initio coordinates of the equilibrium structure of ${ }^{13} \mathrm{CC}_{5} \mathrm{H}_{6}-\mathrm{H}_{2} \mathrm{O}$ complex at MP2 $/ 6-311++\mathrm{g}(\mathrm{d}, \mathrm{p})$ level, including BSSE CP corrections

Table $\mathrm{S} 4$ : Ab initio coordinates of the equilibrium structure of $\mathrm{C}_{6} \mathrm{H}_{6}-\mathrm{H}_{2}^{18} \mathrm{O}$ complex at MP2 $/ 6-311++\mathrm{g}(\mathrm{d}, \mathrm{p})$ level, including BSSE CP corrections

Table S5 : Ab initio coordinates of the equilibrium structure of $\mathrm{Ne}-\mathrm{C}_{6} \mathrm{H}_{6}-\mathrm{H}_{2} \mathrm{O}$ complex at MP2 $/ 6-311++\mathrm{g}(\mathrm{d}, \mathrm{p})$ level, including BSSE CP corrections

Table S6 : Ab initio coordinates of the equilibrium structure of $\mathrm{Ar}-\mathrm{C}_{6} \mathrm{H}_{6}-\mathrm{H}_{2} \mathrm{O}$ complex at MP2 $/ 6-311++\mathrm{g}(\mathrm{d}, \mathrm{p})$ level, including BSSE CP corrections

Table S7 : Ab initio coordinates of the equilibrium structure of $\mathrm{Kr}-\mathrm{C}_{6} \mathrm{H}_{6}-\mathrm{H}_{2} \mathrm{O}$ complex at MP2 $/ 6-311++\mathrm{g}(\mathrm{d}, \mathrm{p})$ level, including BSSE CP corrections

\footnotetext{
${ }^{*}$ Corresponding authors

Email addresses: mangal@iitm.ac.in (Mangala Sunder Krishnan), arunan@iisc.ac.in (E. Arunan)
}

Preprint submitted to Journal of Molecular Spectroscopy

March 23, 2020 
Table S1: Vibrational frequencies from ab initio calculations for benzene-water and rare gas-benzene-water complexes at $\mathrm{MP} 2 / 6-311++\mathrm{g}(\mathrm{d}, \mathrm{p})$ level, including BSSE CP (basis-set superposition error counterpoise) corrections

\begin{tabular}{|c|c|c|c|c|c|c|}
\hline & $\mathrm{C}_{6} \mathrm{H}_{6}-\mathrm{H}_{2} \mathrm{O}$ & ${ }^{13} \mathrm{CC}_{5} \mathrm{H}_{6}-\mathrm{H}_{2} \mathrm{O}$ & $\mathrm{C}_{6} \mathrm{H}_{6}-\mathrm{H}_{2}^{18} \mathrm{O}$ & $\mathrm{Ne}-\mathrm{C}_{6} \mathrm{H}_{6}-\mathrm{H}_{2} \mathrm{O}$ & $\mathrm{Ar}-\mathrm{C}_{6} \mathrm{H}_{6}-\mathrm{H}_{2} \mathrm{O}$ & $\mathrm{Kr}-\mathrm{C}_{6} \mathrm{H}_{6}-\mathrm{H}_{2} \mathrm{O}$ \\
\hline 1 & 12.79 & 12.81 & 12.70 & 9.64 & 10.92 & 11.27 \\
\hline 2 & 28.91 & 28.78 & 28.41 & 10.66 & 15.38 & 17.50 \\
\hline 3 & 42.66 & 42.64 & 42.45 & 17.45 & 21.96 & 25.88 \\
\hline 4 & 77.47 & 77.14 & 74.96 & 20.27 & 28.60 & 33.30 \\
\hline 5 & 107.04 & 107.04 & 106.23 & 31.11 & 34.88 & 40.12 \\
\hline 6 & 228.69 & 228.70 & 227.76 & 44.52 & 46.50 & 49.98 \\
\hline 7 & 388.18 & 385.11 & 388.19 & 78.10 & 78.61 & 79.27 \\
\hline 8 & 388.98 & 388.24 & 388.98 & 107.11 & 106.83 & 105.71 \\
\hline 9 & 609.34 & 603.06 & 609.34 & 230.74 & 230.33 & 229.88 \\
\hline 10 & 609.54 & 608.18 & 609.54 & 388.43 & 387.25 & 386.69 \\
\hline 11 & 682.29 & 681.94 & 682.29 & 389.22 & 388.13 & 387.56 \\
\hline 12 & 742.74 & 739.52 & 742.74 & 609.31 & 609.16 & 609.02 \\
\hline 13 & 856.10 & 855.21 & 856.09 & 609.51 & 609.37 & 609.24 \\
\hline 14 & 857.56 & 856.17 & 857.56 & 682.73 & 682.09 & 681.89 \\
\hline 15 & 959.94 & 956.63 & 959.94 & 742.91 & 741.57 & 740.67 \\
\hline 16 & 960.72 & 960.70 & 960.72 & 856.19 & 855.30 & 855.17 \\
\hline 17 & 1007.57 & 997.19 & 1007.57 & 857.92 & 857.37 & 857.37 \\
\hline 18 & 1014.36 & 1011.74 & 1014.36 & 960.00 & 958.36 & 958.29 \\
\hline 19 & 1059.91 & 1056.66 & 1059.91 & 960.70 & 958.72 & 958.86 \\
\hline 20 & 1060.43 & 1058.13 & 1060.43 & 1007.69 & 1007.66 & 1007.63 \\
\hline 21 & 1079.41 & 1077.04 & 1079.41 & 1014.13 & 1013.94 & 1013.96 \\
\hline 22 & 1173.80 & 1173.58 & 1173.80 & 1059.98 & 1059.92 & 1059.88 \\
\hline 23 & 1199.71 & 1197.69 & 1199.71 & 1060.51 & 1060.46 & 1060.42 \\
\hline 24 & 1200.28 & 1200.25 & 1200.28 & 1078.81 & 1077.24 & 1076.17 \\
\hline 25 & 1374.58 & 1372.20 & 1374.58 & 1173.86 & 1173.78 & 1173.77 \\
\hline 26 & 1447.13 & 1436.28 & 1447.13 & 1199.74 & 1199.64 & 1199.59 \\
\hline 27 & 1505.39 & 1498.75 & 1505.39 & 1200.34 & 1200.24 & 1200.18 \\
\hline 28 & 1505.76 & 1502.44 & 1505.76 & 1374.53 & 1374.38 & 1374.25 \\
\hline 29 & 1634.11 & 1620.42 & 1634.09 & 1447.53 & 1448.38 & 1449.78 \\
\hline 30 & 1634.89 & 1631.85 & 1634.83 & 1505.49 & 1505.39 & 1505.28 \\
\hline 31 & 1644.33 & 1644.31 & 1637.76 & 1505.86 & 1505.76 & 1505.66 \\
\hline 32 & 3198.11 & 3195.48 & 3198.11 & 1634.25 & 1634.06 & 1633.83 \\
\hline 33 & 3207.66 & 3203.97 & 3207.66 & 1635.04 & 1634.85 & 1634.60 \\
\hline 34 & 3209.19 & 3209.15 & 3209.19 & 1644.96 & 1644.80 & 1644.71 \\
\hline 35 & 3223.53 & 3220.78 & 3223.53 & 3197.80 & 3197.83 & 3197.71 \\
\hline 36 & 3225.07 & 3225.00 & 3225.07 & 3207.33 & 3207.34 & 3207.18 \\
\hline 37 & 3233.65 & 3232.40 & 3233.65 & 3208.95 & 3208.96 & 3208.82 \\
\hline 38 & 3868.32 & 3868.32 & 3859.58 & 3223.24 & 3223.23 & 3223.05 \\
\hline 39 & 3980.51 & 3980.51 & 3964.87 & 3224.80 & 3224.80 & 3224.64 \\
\hline 40 & & & & 3233.38 & 3233.36 & 3233.17 \\
\hline 41 & & & & 3868.26 & 3868.25 & 3868.32 \\
\hline 42 & & & & 3980.04 & 3980.04 & 3980.05 \\
\hline
\end{tabular}


Table S2: $A b$ initio coordinates of the equilibrium structure of $\mathrm{C}_{6} \mathrm{H}_{6}-\mathrm{H}_{2} \mathrm{O}$ complex at MP2/6$311++\mathrm{g}(\mathrm{d}, \mathrm{p})$ level, including BSSE CP corrections

\begin{tabular}{cccc}
\hline Atom & $\mathrm{X} / \AA$ & $\mathrm{Y} / \AA$ & $\mathrm{Z} / \AA$ \\
\hline $\mathrm{C}$ & 0.371473 & 0.950480 & -1.084740 \\
$\mathrm{C}$ & 0.577476 & -0.407225 & -1.363890 \\
$\mathrm{C}$ & 0.829698 & -1.305537 & -0.319368 \\
$\mathrm{C}$ & 0.941632 & -0.836183 & 0.995915 \\
$\mathrm{C}$ & 0.734773 & 0.520684 & 1.275504 \\
$\mathrm{C}$ & 0.482993 & 1.419679 & 0.231149 \\
$\mathrm{H}$ & 0.495551 & -0.770030 & -2.384734 \\
$\mathrm{H}$ & 0.985673 & -2.358969 & -0.535599 \\
$\mathrm{H}$ & 1.147162 & -1.531905 & 1.805139 \\
$\mathrm{H}$ & 0.815291 & 0.883828 & 2.296388 \\
$\mathrm{H}$ & 0.329060 & 2.473426 & 0.446888 \\
$\mathrm{H}$ & 0.173658 & 1.647319 & -1.894802 \\
$\mathrm{H}$ & -2.575221 & -0.932310 & 0.713372 \\
$\mathrm{H}$ & -2.040903 & 0.182337 & -0.135352 \\
$\mathrm{O}$ & -2.869818 & -0.205635 & 0.160159 \\
\hline
\end{tabular}

Table S3: $A b$ initio coordinates of the equilibrium structure of ${ }^{13} \mathrm{CC}_{5} \mathrm{H}_{6}-\mathrm{H}_{2} \mathrm{O}$ complex at MP2/6$311++\mathrm{g}(\mathrm{d}, \mathrm{p})$ level, including BSSE CP corrections

\begin{tabular}{cccc}
\hline Atom & $\mathrm{X} / \AA$ & $\mathrm{Y} / \AA$ & $\mathrm{Z} / \AA$ \\
\hline${ }^{13} \mathrm{C}$ & -0.371473 & 0.950480 & 1.084740 \\
$\mathrm{C}$ & -0.577476 & -0.407225 & 1.363890 \\
$\mathrm{C}$ & -0.829698 & -1.305537 & 0.319368 \\
$\mathrm{C}$ & -0.941632 & -0.836183 & -0.995915 \\
$\mathrm{C}$ & -0.734773 & 0.520684 & -1.275504 \\
$\mathrm{C}$ & -0.482993 & 1.419679 & -0.231149 \\
$\mathrm{H}$ & -0.495552 & -0.770030 & 2.384734 \\
$\mathrm{H}$ & -0.985673 & -2.358969 & 0.535599 \\
$\mathrm{H}$ & -1.147162 & -1.531905 & -1.805139 \\
$\mathrm{H}$ & -0.815291 & 0.883828 & -2.296389 \\
$\mathrm{H}$ & -0.329060 & 2.473426 & -0.446888 \\
$\mathrm{H}$ & -0.173658 & 1.647319 & 1.894802 \\
$\mathrm{H}$ & 2.575221 & -0.932310 & -0.713372 \\
$\mathrm{H}$ & 2.040903 & 0.182337 & 0.135352 \\
$\mathrm{O}$ & 2.869818 & -0.205635 & -0.160159 \\
\hline
\end{tabular}


Table S4: $A b$ initio coordinates of the equilibrium structure of $\mathrm{C}_{6} \mathrm{H}_{6}-\mathrm{H}_{2}^{18} \mathrm{O}$ complex at MP2/6$311++\mathrm{g}(\mathrm{d}, \mathrm{p})$ level, including BSSE CP corrections

\begin{tabular}{cccc}
\hline Atom & $\mathrm{X} / \AA$ & $\mathrm{Y} / \AA$ & $\mathrm{Z} / \AA$ \\
\hline $\mathrm{C}$ & -0.371473 & 0.950480 & 1.084740 \\
$\mathrm{C}$ & -0.577476 & -0.407225 & 1.363890 \\
$\mathrm{C}$ & -0.829698 & -1.305537 & 0.319368 \\
$\mathrm{C}$ & -0.941632 & -0.836183 & -0.995915 \\
$\mathrm{C}$ & -0.734773 & 0.520684 & -1.275504 \\
$\mathrm{C}$ & -0.482993 & 1.419679 & -0.231149 \\
$\mathrm{H}$ & -0.495552 & -0.770030 & 2.384734 \\
$\mathrm{H}$ & -0.985673 & -2.358969 & 0.535599 \\
$\mathrm{H}$ & -1.147162 & -1.531905 & -1.805139 \\
$\mathrm{H}$ & -0.815291 & 0.883828 & -2.296389 \\
$\mathrm{H}$ & -0.329060 & 2.473426 & -0.446888 \\
$\mathrm{H}$ & -0.173658 & 1.647319 & 1.894802 \\
$\mathrm{H}$ & 2.575221 & -0.932310 & -0.713372 \\
$\mathrm{H}$ & 2.040903 & 0.182337 & 0.135352 \\
${ }^{18} \mathrm{O}$ & 2.869818 & -0.205635 & -0.160159 \\
\hline
\end{tabular}

Table S5: $A b$ initio coordinates of the equilibrium structure of $\mathrm{Ne}-\mathrm{C}_{6} \mathrm{H}_{6}-\mathrm{H}_{2} \mathrm{O}$ complex at MP2/6$311++\mathrm{g}(\mathrm{d}, \mathrm{p})$ level, including BSSE CP corrections

\begin{tabular}{cccc}
\hline Atom & $\mathrm{X} / \AA$ & $\mathrm{Y} / \AA$ & $\mathrm{Z} / \AA$ \\
\hline $\mathrm{C}$ & -0.128491 & -1.324785 & 0.218031 \\
$\mathrm{C}$ & -0.076518 & -0.446912 & 1.309030 \\
$\mathrm{C}$ & -0.043449 & 0.936446 & 1.092771 \\
$\mathrm{C}$ & 0.004820 & 1.439487 & -0.213844 \\
$\mathrm{C}$ & -0.048361 & 0.562416 & -1.304710 \\
$\mathrm{C}$ & -0.081336 & -0.821164 & -1.088900 \\
$\mathrm{H}$ & -0.109035 & -0.837767 & 2.322318 \\
$\mathrm{H}$ & -0.008132 & 1.617198 & 1.939000 \\
$\mathrm{H}$ & 0.040188 & 2.512728 & -0.381555 \\
$\mathrm{H}$ & -0.017882 & 0.952860 & -2.318246 \\
$\mathrm{H}$ & -0.116239 & -1.502104 & -1.934930 \\
$\mathrm{H}$ & -0.156216 & -2.398140 & 0.385733 \\
$\mathrm{H}$ & -3.420935 & 0.786149 & -0.028603 \\
$\mathrm{H}$ & -2.697727 & -0.526429 & 0.006705 \\
$\mathrm{O}$ & -3.586382 & -0.158881 & -0.006664 \\
$\mathrm{Ne}$ & 3.741705 & -0.140637 & -0.001138 \\
\hline
\end{tabular}


Table S6: $A b$ initio coordinates of the equilibrium structure of $\mathrm{Ar}-\mathrm{C}_{6} \mathrm{H}_{6}-\mathrm{H}_{2} \mathrm{O}$ complex at MP2/6$311++\mathrm{g}(\mathrm{d}, \mathrm{p})$ level, including BSSE CP corrections

\begin{tabular}{cccc}
\hline Atom & $\mathrm{X} / \AA$ & $\mathrm{Y} / \AA$ & $\mathrm{Z} / \AA$ \\
\hline $\mathrm{C}$ & -0.540151 & -1.315270 & 0.218834 \\
$\mathrm{C}$ & -0.498984 & -0.436226 & 1.309352 \\
$\mathrm{C}$ & -0.482749 & 0.947312 & 1.092331 \\
$\mathrm{C}$ & -0.440484 & 1.450182 & -0.214558 \\
$\mathrm{C}$ & -0.482869 & 0.571926 & -1.304943 \\
$\mathrm{C}$ & -0.499006 & -0.811833 & -1.088371 \\
$\mathrm{H}$ & -0.526836 & -0.826889 & 2.322853 \\
$\mathrm{H}$ & -0.455812 & 1.628911 & 1.938186 \\
$\mathrm{H}$ & -0.418177 & 2.523682 & -0.382859 \\
$\mathrm{H}$ & -0.457049 & 0.962153 & -2.318692 \\
$\mathrm{H}$ & -0.525528 & -1.493615 & -1.934027 \\
$\mathrm{H}$ & -0.554813 & -2.388790 & 0.387128 \\
$\mathrm{H}$ & -3.858043 & 0.755259 & -0.029269 \\
$\mathrm{H}$ & -3.118901 & -0.548391 & 0.006831 \\
$\mathrm{O}$ & -4.011967 & -0.191704 & -0.006822 \\
$\mathrm{Ar}$ & 3.315353 & -0.084180 & -0.000636 \\
\hline
\end{tabular}

Table S7: $A b$ initio coordinates of the equilibrium structure of $\mathrm{Kr}-\mathrm{C}_{6} \mathrm{H}_{6}-\mathrm{H}_{2} \mathrm{O}$ complex at MP2/6$311++\mathrm{g}(\mathrm{d}, \mathrm{p})$ level, including BSSE CP corrections

\begin{tabular}{cccc}
\hline Atom & $\mathrm{X} / \AA$ & $\mathrm{Y} / \AA$ & $\mathrm{Z} / \AA$ \\
\hline $\mathrm{C}$ & -1.186679 & -1.312101 & 0.204664 \\
$\mathrm{C}$ & -1.150380 & -0.443305 & 1.303516 \\
$\mathrm{C}$ & -1.143789 & 0.942332 & 1.099761 \\
$\mathrm{C}$ & -1.105977 & 1.457974 & -0.202279 \\
$\mathrm{C}$ & -1.143451 & 0.589966 & -1.301014 \\
$\mathrm{C}$ & -1.149839 & -0.795886 & -1.097675 \\
$\mathrm{H}$ & -1.174551 & -0.843818 & 2.313271 \\
$\mathrm{H}$ & -1.120490 & 1.615963 & 1.952119 \\
$\mathrm{H}$ & -1.091149 & 2.533189 & -0.360258 \\
$\mathrm{H}$ & -1.120608 & 0.990012 & -2.311023 \\
$\mathrm{H}$ & -1.172413 & -1.469730 & -1.949813 \\
$\mathrm{H}$ & -1.193617 & -2.387292 & 0.362640 \\
$\mathrm{H}$ & -4.517851 & 0.739660 & -0.018209 \\
$\mathrm{H}$ & -3.772379 & -0.560670 & 0.004220 \\
$\mathrm{O}$ & -4.667173 & -0.208208 & -0.004355 \\
$\mathrm{Kr}$ & 2.605031 & -0.044043 & 0.000002 \\
\hline
\end{tabular}

